\title{
Determination of veterinary pharmaceuticals residue in soil and biological materials: a review of current analytical methods
}

\begin{abstract}
Veterinary pharmaceuticals have been extensively used in animal husbandry for control of disease and growth promoters. These compounds are excreted from animals via urine and faeces, end up in the environment through untreated animal waste disposal. Veterinary pharmaceuticals often exist in the complex solid environmental samples such as manure, slurry, and soil which require extensive extraction, clean-up and analysis method. This review highlights the current analytical methods for the analysis of veterinary pharmaceuticals in complex solid environmental matrices, including soil, animal manures and sediment. The aim of this review is to compare and summarize the performance of each method in terms of recovery, method detection limit (MDL) and method quantification limit (MQL).
\end{abstract}

Keyword: Review; Veterinary pharmaceuticals; Soil; Manure; Analytical methods 